\title{
Exploiting sensor redistribution for eliminating the energy hole problem in mobile sensor networks
}

\author{
Jie Jia*, Xueli Wu, Jian Chen and Xingwei Wang
}

\begin{abstract}
The use of mobile sensors is of great relevance to monitor hazardous applications where sensors cannot be deployed manually. Traditional algorithms primarily aim at maximizing network coverage rate, which leads to the creation of the "energy hole" in the region near the sink node. In this article, we are addressing the problem of redistributing mobile sensor nodes over an unattended target area. Driven by energy efficiency considerations, a pixel-based transmission scheme is developed to reduce extra overhead caused by frequent sensing and decision making. We derive the optimal node distribution and provide a theoretical explanation of balanced energy depletion for corona-based sensor network. In addition, we demonstrate that it can be extended to deal with uneven energy depletion due to the many-to-one communications in multi-hop wireless sensor networks. Applying the optimal condition, we then propose a novel sensor redistribution algorithm to completely eliminate the energy hole problem in mobile sensor network. Extensive simulation results verify that the proposed solution outperforms others in terms of coverage rate, average moving distance, residual energy, and total energy consumption.
\end{abstract}

\section{Introduction}

Wireless sensor network (WSN) usually consists of a large number of static sensor nodes that are densely deployed for object monitoring and target tracking either inside the phenomenon or very close to it [1]. Sensor nodes are able to measure various parameters of the environment and transmit collected data to the sink node through multi-hop communication. Once the sink node received sensed data, it processes and forwards it to the users.

Nodes deployment is the first step in establishing a sensor network. In general, sensor nodes are typically battery powered and randomly deployed over a target area. Once deployed, they are left unattended. In many potential working environments, such as monitoring hazardous applications over disaster areas, deploying such a stationary sensor network cannot be performed manually or precisely. Thus, maintaining its sensing coverage could be a difficult task. As a result, it is necessary

\footnotetext{
* Correspondence: jiajie@ise.neu.edu.cn

College of Information Science and Engineering, Northeastern University,
} Shenyang, China

\section{Springer

(C) $2012 \mathrm{Jia}$ et al; licensee Springer. This is an Open Access article distributed under the terms of the Creative Commons Attribution License (http://creativecommons.org/licenses/by/2.0), which permits unrestricted use, distribution, and reproduction in any medium, provided the original work is properly cited. to make use of mobile sensors, which can autonomously discover and repair coverage holes.

How to optimize energy consumption to prolong network lifetime is one of the fundamental issues arising in WSN. To address this issue, much work has been done during recent years where mobility of sensors is taken advantage of to achieve desired distribution [2-8]. Typically, most of these works have addressed the redistribution of mobile sensors to achieve a uniform coverage of a certain density in the target area. If the sensor nodes are deployed uniformly, the sensors closer to the sink not only need to send their own sensed data, but also forward data collected by other sensors farther away from the sink node. In this case, those sensors near the sink node will consume more energy and die more quickly. Once those nodes are dead, no more data can be transmitted to the sink. As a result, the network would get disconnected, with up to $90 \%$ of the total initial energy left unused in a normal uniform distribution [9]. Exploiting redistribution of the nodes by using sensor mobility to balance the energy depletion is of great importance to prolong the network lifetime. 
In this article, we investigate and try to eliminate the energy hole problem with non-uniform node distribution in mobile WSN. We first propose a new data transmission mechanism to reduce the redundant messages being sent. We also prove that based on this transmission mechanism, an energy-balanced depletion among all the working sensors is possible when each corona has an appropriate node density. Then, the concept of the equivalent sensing radius is devised and a novel sensor distribution algorithm for mobile sensor networks is proposed to achieve balanced energy depletion based on genetic algorithm. Further, we conduct extensive simulations to validate the analysis and compare the performance of these algorithms. Simulation results show that when the network lifetime ends, the nodes in the target area almost use up their energy simultaneously, which can prolong the network lifetime effectively.

\section{Related study}

Sensor distribution is a critical issue because it affects the cost, connectivity, and detection capability of WSN. There has been some work on the sensor distribution to maintain full coverage as well as connectivity with optimal sensor movement for mobile sensor networks. In [4], the authors assume that there are virtual attractive and repulsive forces among sensors, and based on these virtual forces, sensors can spread throughout the environment with a uniform distribution to achieve the network coverage. In [6], the authors propose a Voronoi diagram-based distribution model, in which each sensor iteratively calculates its Voronoi polygon to detect its coverage holes and moves to a better position to enhance the coverage rate of the field. In [7], the authors investigate how to move sensors while still maintaining complete coverage of the field. In [8], the sensing field is divided into grids. And then, the sensors move from high-density grids to low-density ones to construct a uniform topology. These algorithms all focused on finding a uniform distribution of sensor nodes, to improve the coverage performance for mobile sensor network. However, as the uniform distribution may lead to unbalanced energy depletion, the above approaches will cause prematurely the end of the network lifetime with a considerable amount of energy wasted.

The problem of uneven energy consumption in a large class of many-to-one sensor networks was investigated by Li and Mohapatra [9] for the first time. Further, they proposed several approaches to mitigate this problem and inferred that simply increasing the number of nodes cannot prolong the system lifetime under a uniform distribution [10]. In [11], the authors propose a transmission range adjustment approach to tackle the unbalanced energy depletion. However, searching the optimal transmission ranges of sensors among all the coronas is an NP-complete problem. In [12], the authors use mobile sensors to heal energy holes, but the cost of their approaches is considerably large. The mobile relays [13] and mobile sink [14] are also imported to avoid energy hole. However, as the nodes near the sink or relay nodes always changed over time, the energy imbalance is only mitigated and how to plan the optimal mobility trajectory is very difficult. In [15], the authors focus on variable node distribution density in order to mitigate the effects of the uneven energy depletion. However, associated with their routing strategy, the uneven energy depletion still exists. The authors in [16] also investigated the energy hole problem in WSN with non-uniform node distribution. With their theoretical analysis, when all the sensors have a constant data acquisition rate, the energybalanced depletion among the whole network is impossible. Nevertheless, nearly balanced energy depletion in the network is possible if the number of nodes increases in geometric progression from the outer coronas to the inner ones except the outermost one. Based on this strategy, the authors in [17] propose an autonomous sensor redeployment algorithm $\delta$-Push\&Pull to mitigate the sink-hole problem. However, as they assume that each sensor has a constant data acquisition rate, which may not be true for highly dense WSN and the uneven energy depletion still exists between the outermost corona and the inner coronas. In fact, we can prove that completely balanced energy depletion is achievable with the additional help of pixel-based transmission mechanism in this article.

The rest of the article is organized as follows. Section 3 describes the preliminary work and the network model for our discussion. Section 4 theoretically analyzes how to balance the energy depletion and computes the node density for each corona. A new non-uniform node distribution strategy is proposed for energybalanced depletion in Section 5. Section 6 presents the simulation results for our algorithms, and Section 7 concludes the article.

\section{Preliminary work and network model \\ 3.1. Network model and assumptions}

In this section, we present our network model and make some basic assumptions about such a model. Assume that a set of $N$ heterogeneous sensors are deployed in a circular area with radius $d$ in order to monitor some physical phenomenon. Each sensor node has an ID, a fixed transmission range $R_{c}$, and a fixed sensing range $R_{s}$. All of the sensor nodes are aware of their position and can report the location where the information is sensed. The only sink node is located at the centre of the circle, as shown in Figure 1. 


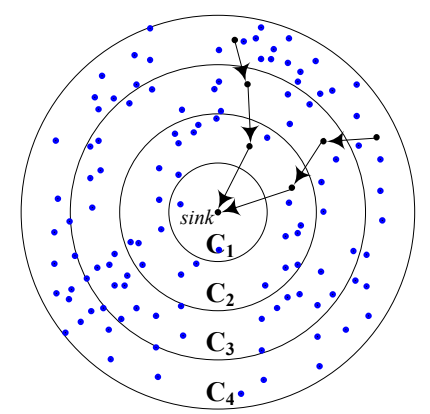

Figure $1 \mathrm{~A}$ circular target area with four coronas.

We divide the area into $n$ adjacent coronas with the same width of $R_{c}$. For clear presentation, the coronas from inside to outside are denoted as $C_{1}, C_{2}, \ldots, C_{i}, \ldots, C_{n}$. Obviously, the corona $C_{i}$ is composed of nodes whose distances to the sink are between $(i-1)^{*} R_{c}$ and $i^{*} R_{c}$.

The network works in two phases: the first phase of node redistribution and the second phase of field monitoring and data gathering. During the second phase, each working sensor should send its sensing message to the sink node periodically. The corona survival lifetime is defined as the number of working rounds in which its sensors participate until the first sensor runs out of energy. With regard to the network survival lifetime, it can be calculated as the minimum survival time of its coronas.

We use a simplified power consumption model and do not mention any physical layer functionality or solution in MAC layer. In our model, the energy consumption is only dominated by communication costs, as opposed to sensing and processing costs. The initial energy of each sensor is set as $\varepsilon>0$, and the sink node has no energy limitation. We further assume that a sensor consumes $e_{1}$ units of energy when sending one bit while it depletes $e_{2}$ units of energy when receiving one bit, where $e_{1}>e_{2}>0$.

\subsection{Coverage model}

The target area $A$ is digitized into $m^{*} n$ pixels and each pixel size is equal to 1 . We refer to the set of sensor nodes which has been deployed in the target area as $S=$ $\left\{s_{1}, s_{2}, \ldots, s_{N}\right\}$. The coverage model of each sensor $s_{i}$ can be expressed as a circle with center of its coordinates $\left(x_{i}, y_{i}\right)$ and radius $r_{i}$. A random variable $c_{i}$ is introduced to describe the event that a pixel $(x, y)$ is covered by the sensor $s_{i}$. In hence, the probability of an event $c_{i}$, denoted as $P\left\{c_{i}\right\}$, is equal to the coverage probability $P_{\text {cov }}\left(x, y, n_{i}\right)$. This may degenerate to a two-valued function

$$
P\left\{c_{i}\right\}=P_{\operatorname{cov}}\left(x, y, s_{i}\right)=\left\{\begin{array}{l}
1 \text { if }\left(x-x_{i}\right)^{2}+\left(y-y_{i}\right)^{2} \leq r_{i}^{2} \\
0 \quad \text { otherwise. }
\end{array}\right.
$$

That is to say, a pixel $(x, y)$ is covered by a sensor $s_{i}$ if its distance to the circle center $\left(x_{i}, y_{i}\right)$ is not larger than the radius $r_{i}$. Since any random event $c_{i}$ is independent to the others, $r_{i}$ and $r_{j}$ are unrelated, $i, j \in[1, N]$ and $i$ $\neq j$. Then, the following two relationships can be concluded,

$$
\left\{\begin{array}{c}
P\left\{\bar{c}_{i}\right\}=1-P\left\{c_{i}\right\}=1-P_{\mathrm{cov}}\left(x, y, n_{i}\right) \\
P\left\{c_{i} \cup c_{j}\right\}=1-P\left\{\bar{c}_{i} \cap \bar{c}_{j}\right\}=1-P\left\{\bar{c}_{i}\right\} \cdot P\left\{\bar{c}_{j}\right\}
\end{array}\right.
$$

where $\bar{c}_{i}$ is the complement of $c_{i}$, denoting that $s_{i}$ fails to cover the pixel $(x, y)$. It can be considered that the pixel $(x, y)$ is covered if any node in the set covers it. So, the probability of the pixel $(x, y)$ covered by the node set can be denoted as the union of $c_{i}$

$$
\begin{aligned}
P_{\text {cov }}(x, y, S) & =P\left\{\bigcup_{i=1}^{N} c_{i}\right\}=1-P\left\{\bigcap_{i=1}^{N} \bar{c}_{i}\right\} \\
& =1-\prod_{i=1}^{N}\left(1-P_{\operatorname{cov}}\left(x, y, n_{i}\right)\right) .
\end{aligned}
$$

Finally, we define the coverage rate of the sensor set $P_{\text {cov }}(S)$ as the proportion of the coverage area $A_{\text {area }}(C)$ to the total area $A_{s}$,

$$
P_{\mathrm{COV}}(S)=\sum_{x=1}^{m} \sum_{y=1}^{n} P_{\mathrm{Cov}}(x, y, C) /(m \times n)
$$

\subsection{Pixel-based transmission mechanism}

With traditional transmission mechanism, the sensing messages for redundant coverage area would be retransmitted by more than one sensor, causing a tremendous amount of energy to be wasted [15]. In order to save energy, a novel data transmission mechanism is deigned in this article.

After all the sensor nodes are redistributed, each sensor node needs to decide its working area for periodically field sensing and transmitting. In this article, we take intersection of each sensor's Voronoi polygon and its sensing disk as the sensing area, as shown in Figure 2. The Voronoi polygon is an important graph in computational geometry $[18,19]$, which can represent the proximity information of a set of geometric nodes. Since the interior pixels in a given polygon are closer to its sensor than to any other one, with the help of Voronoi polygon, it can guarantee that the sensing message for each pixel is sensed and transmitted only once.

To construct the Voronoi polygon, all the sensors should calculate the bisectors of their neighbors and themselves. These bisectors could form several polygons, and the smallest one encircling the sensor is the Voronoi polygon of this sensor. In our approach, as all the 


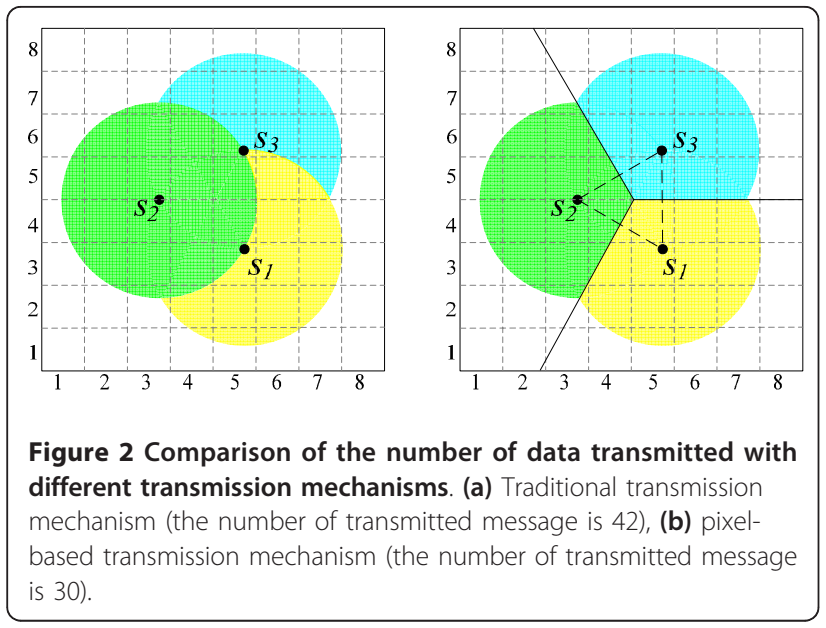

sensors keep stationary after redistribution, the Voronoi graph is constructed only when the process of nodes redistribution control has finished. Hence, the Voronoi diagram will remain unchanged until the end of network lifetime. Furthermore, in order to reduce the extra overhead caused by frequent sensing area decision and minimize the sensing time efficiently, each node should remember all of the sensing pixels after its Voronoi polygon is built. With these supports, the extra energy consumption caused by sensing area decision is similar to the typical Voronoi application in WSNs [6]. By the use of Voronoi polygon construction and pixel remembering, this is named as pixel-based transmission mechanism in this article.

Figure 2 shows the difference of our mechanism with traditional mechanism, where Figure $2 \mathrm{a}$ is the initial sensor deployment, and Figure $2 \mathrm{~b}$ is the corresponding sensing area in each sensor's Voionoi diagram (with different colors). When traditional transmission mechanism is used, the number of messages transmitted by sensor $S_{2}$ is 14 (the green area shown in Figure 2a). While the pixel-based transmission mechanism is used, the number of messages transmitted reduced to 10 . To sum up, as the duplicate sensing message is sent only once by the use of pixelbased transmission mechanism, the total number of duplicated messages saved to transmit is 12 .

\section{Accessibility condition for energy-balanced depletion}

In our network model, nodes belonging to corona $\left\{C_{i} \mid i\right.$ $\neq n$ \} will forward both the data generated by themselves and the data generated by coronas $\left\{C_{j} \mid(i+1) \leq j \leq n\right\}$. While the nodes in the outermost corona $C_{n}$ need not forward any data. Assume that the sensors in each corona are distributed uniformly and there is no data aggregation at any forwarding nodes. Define the number of nodes deployed in corona $C_{i}$ is $N_{i}$ and the number of pixels in corona $C_{i}$ is $A_{i}$. Based on the pixel-based transmission mechanism, the number of messages for corona $C_{i}$ to receive and forward is $\left(A_{i+1}+A_{i+2}+\cdots+A_{n}\right)$ and $\left(A_{i}\right.$ $\left.+A_{i+1}+\cdots+A_{n}\right)$. As the sensing messages are transmitted per working round, the average energy consumption per working round of sensors in corona $C_{i}$ is

$$
\bar{E}_{i}=\left[\sum_{k=i+1}^{n} A_{k}\left(e_{1}+e_{2}\right)+A_{i} e_{1}\right] / N_{i}, 1 \leq i \leq n-1 .
$$

where $e_{1}$ is the energy consumed in sending one bit message, and $e_{2}$ is the energy consumed in receiving one bit message. Note that Equation (5) can be simplified as

$$
\bar{E}_{i}=\frac{e_{1}}{\rho_{i}}+\frac{A_{i+1}+A_{i+2}+\cdots+A_{n}}{A_{i} \cdot \rho_{i}}\left(e_{1}+e_{2}\right), 1 \leq i \leq n-1 .
$$

where $\rho_{i}$ is the node density of corona $C_{i}$.

Sensors in corona $C_{n}$ only need to send their own sensing messages, so the energy depletion of sensors in corona $C_{n}$ is

$$
\bar{E}_{n}=A_{n} \cdot e_{1} / N_{n}=e_{1} / \rho_{n}
$$

Thus, we can formulate $\bar{E}_{i}$ as follows

$$
\bar{E}_{i}= \begin{cases}e_{1} / \rho_{n,} & i=n, \\ \frac{e_{1}}{\rho_{i}}+\frac{\sum_{k=i+1}^{n} A_{k}}{A_{i} \cdot \rho_{i}}\left(e_{1}+e_{2}\right) & , 1 \leq i \leq n-1 .\end{cases}
$$

Ideally, when all the nodes deplete their energy with the same ratio, the network lifetime is prolonged and the energy efficiency is improved. In particular, there is no energy wasted and the network lifetime can be given by

$$
\frac{\varepsilon}{\overline{\bar{E}}_{1}}=\frac{\varepsilon}{\bar{E}_{2}}=\cdots=\frac{\varepsilon}{\overline{\bar{E}}_{i}}=\cdots=\frac{\varepsilon}{\overline{\bar{E}}_{n}}
$$

Theorem 1: Perfect and maximum energy efficiency is possible when the node distribution density $\rho_{i}$ in corona $C_{i}$ satisfies

$$
\rho_{i}=\rho_{n} \cdot\left[1+\frac{\left(n^{2}-i^{2}\right) \cdot\left(e_{1}+e_{2}\right)}{(2 i-1) \cdot e_{1}}\right], \rho_{1} \geq \rho_{2} \geq \cdots \geq \rho_{n}
$$

Proof: To use the deductive method, suppose Equation (10) is true, thus Equation (6) can be rewrote as follows

$$
\begin{aligned}
\bar{E}_{i} & =\frac{A_{i} \cdot e_{1}+\sum_{i=k+1}^{n} A_{i} \cdot\left(e_{1}+e_{2}\right)}{A_{i} \cdot \rho_{i}} \\
& =\frac{\left[A_{i} \cdot e_{1}+\sum_{i=k+1}^{n} A_{i} \cdot\left(e_{1}+e_{2}\right)\right] \cdot(2 i-1) \cdot e_{1}}{A_{i} \cdot \rho_{n} \cdot\left[(2 i-1) \cdot e_{1}+\left(n^{2}-i^{2}\right) \cdot\left(e_{1}+e_{2}\right)\right]}
\end{aligned}
$$


Owing to $A_{i}=\pi R_{c}^{2} \cdot(2 i-1)$, after basic transformations, we have

$$
\begin{aligned}
\bar{E}_{i} & =\frac{\left[(2 i-1) \cdot e_{1}+\left(n^{2}-i^{2}\right) \cdot\left(e_{1}+e_{2}\right)\right] \cdot(2 i-1) \cdot e_{1}}{(2 i-1) \cdot \rho_{n} \cdot\left[(2 i-1) \cdot e_{1}+\left(n^{2}-i^{2}\right) \cdot\left(e_{1}+e_{2}\right)\right]} \\
& =\frac{e_{1}}{\rho_{n}}=\bar{E}_{n}
\end{aligned}
$$

Since $\frac{d \rho_{i}}{d i}=\frac{-2 i}{(2 i-1)}-\frac{2\left(n^{2}-i^{2}\right)}{(2 i-1)^{2}}<0$ is a permanent establishment, we can get the following conclusion, $\rho_{1} \geq \rho_{2} \geq \cdots \geq \rho_{n}$. This completes the proof of Theorem 1.

From Theorem 1, if all the sensors adopt the pixelbased data transmission mechanism, and the node density of each corona obeys a certain condition, the energy-balanced depletion of the whole network can be achieved. In addition, we can draw a conclusion that $\rho_{i}$ only relates to $\rho_{n}$ and its corona number $i$.

Further we will analyze the lifetime enhancement of the non-uniform distribution strategy to the traditional one. Note that the node density in non-uniform distribution satisfies Equation (10) and the initial conditions are the same. In the uniform distribution, the density $\rho_{i}$ is equal to $\rho_{n}$. As the innermost corona $C_{1}$ needs to forward all of the sensing messages in the whole network, it consumes the most energy. Thus, the maximum lifetime of network in uniform distribution is determined by the survival time $C_{1}$. The network lifetime can be calculated as

$$
\begin{aligned}
\frac{\varepsilon}{\overline{\bar{E}}_{1}^{\prime}} & =\varepsilon /\left(\frac{e_{1}}{\rho_{n}}+\frac{\sum_{j=2}^{n} A_{j}}{A_{1} \cdot \rho_{n}}\left(e_{1}+e_{2}\right)\right) . \\
& =\frac{\rho_{n} \cdot \varepsilon}{e_{1}+\left(n^{2}-1\right) \cdot\left(e_{1}+e_{2}\right)}
\end{aligned}
$$

where $\bar{E}_{1}^{\prime}$ is the average energy depletion of $C_{1}$ per unit time in uniform distribution. Using Equation (8), we can get the average energy depletion in $C_{1}$ under energy-balanced conditions as

$$
\bar{E}_{1}=\bar{E}_{2}=\cdots \bar{E}_{i}=\cdots=\bar{E}_{n}=\frac{e_{1}}{\rho_{n}} .
$$

Thus, the lifetime enhancement is

$$
\frac{\varepsilon / \bar{E}_{1}}{\varepsilon / \bar{E}_{1}^{\prime}}=\frac{e_{1}+\left(n^{2}-1\right) \cdot\left(e_{1}+e_{2}\right)}{\rho_{n}} / \frac{e_{1}}{\rho_{n}}=\frac{\rho_{1}}{\rho_{n}}>1 .
$$

Therefore, the network lifetime of non-uniform distribution can be extended $\rho_{1} / \rho_{n}$ times effectively compared with the traditional uniform distribution strategy.

\section{Non-uniform node distribution optimization}

In this article, the energy-balanced node distribution is defined as the state when all the working sensors in the whole network use up their energy simultaneously. In this section, we first describe the concept of equivalent sensing radius. And then, the energy-balanced node distribution problem is transformed into uniform distribution optimization problem with different sensing radius. Further, we give an NSGA-II-based node [20] redistribution approach to solve this problem.

\subsection{Equivalent sensing radius}

Definition 1 (equivalent sensing radius): it is defined as the sensing radius when the given distribution density $\rho_{i}$ is the lowest one to maintain network coverage.

As the hexagonal distribution is the optimal sensor distribution to cover the target area completely with the fewest sensors [21], we define $\operatorname{Hex}(i)$ as the hexagonal area covered by sensor $s_{i}$ with the sensing radius $R_{i}$. It can be calculated as

$$
\operatorname{Hex}(i)=\frac{3 \sqrt{3}}{2} R_{i}^{2}
$$

And the minimum distribution density $\rho_{i}$ to fully cover the area is

$$
\rho_{i}=\frac{1}{\operatorname{Hex}(i)} .
$$

Thus, the relationship of the equivalent sensing radius and the optimal distribution density $\rho_{i}$ is

$$
R_{i}=\sqrt{\frac{2}{\sqrt{27} \cdot \rho_{i}}} .
$$

Theorem 2: If the sensor selection algorithm uses the equivalent sensing radius $R_{i}$ according to the density $\rho_{i}$, the network can achieve balanced energy depletion, where $R_{i}$ satisfies,

$$
R_{i}=R_{s} \sqrt{\frac{(2 i-1) \cdot e_{1}}{(2 i-1) \cdot e_{1}+\left(n^{2}-i^{2}\right) \cdot\left(e_{1}+e_{2}\right)}}, R_{1} \leq R_{2} \leq \cdots \leq R_{n} .
$$

Proof: According to Equation (18), the minimum density $\rho_{i}$ to fully cover the corona $C_{i}$ with equivalent sensing radius $R_{i}$ can be calculated as

$$
\rho_{i}=\frac{2}{\sqrt{27} \cdot R_{i}^{2}}
$$

As the outermost corona only needs to send its own sensing messages, its sensing radius is equal to its 
sensing radius $R_{s}$. Then the minimum density $\rho_{s}$ to fully cover the corona $C_{n}$ is

$$
\rho_{s}=\frac{2}{\sqrt{27} \cdot R_{s}^{2}}
$$

Substitute the above equation into Equation (10) and combine it with Equation (20), we have

$$
\begin{aligned}
\rho_{i} & =\frac{2}{\sqrt{27} \cdot R_{i}^{2}} \\
& =\frac{2}{\sqrt{27} \cdot R_{s}{ }^{2}} \cdot\left[1+\frac{\left(n^{2}-i^{2}\right) \cdot\left(e_{1}+e_{2}\right)}{(2 i-1) \cdot e_{1}}\right]
\end{aligned}
$$

After transformation, we have

$$
R_{i}=R_{s} \cdot \sqrt{\frac{(2 i-1) \cdot e_{1}}{(2 i-1) \cdot e_{1}+\left(n^{2}-i^{2}\right) \cdot\left(e_{1}+e_{2}\right)}} .
$$

This concludes the proof of Theorem 2 .

Therefore, by introducing the equivalent sensing radius, this thorny issue can be transformed into a uniform distribution optimization problem with different sensing radius, which gives the chance of using present distribution algorithms. In this article, the node distribution algorithm is combined with our previous NSGA-IIbased approach [22], in which we made major modifications to satisfy the condition defined in Equation (10).

The novel sensor distribution algorithm mainly contains two parts: movement control among different coronas and movement control in each corona. The first part aims at moving the nodes between the adjacent coronas so as to meet the needs of different sensor densities, while the second part aims to achieve an optimal node distribution.

\subsection{Movement control among coronas}

As the nodes are randomly deployed in the target area, this uncertainty may cause that the number of deployed nodes is greater or less than that the corona really needs. The movement control among coronas will satisfy the desired node density according to Equation (10) for each corona. Meanwhile, in order to avoid consuming too much energy in the moving process, the nodes are only allowed moving to the adjacent coronas. By using a stepwise manner, the whole moving process is shown as follows

Step 1: The sink or the cluster head counts sensors deployed for each corona. Set the number of sensors deployed in corona $C_{i}$ is deployed $\mathrm{NumIn} C_{i}$.

Step 2. The sink or the cluster head computes the desired number of sensors desireNumInC $C_{i}$ for each corona. It is calculated as desireNumInC $C_{i}=\rho_{i}{ }^{*} S_{i}$, where $S_{i}$ is the area of corona $C_{i}$.
Step 3. From the outermost corona $C_{N}$ to the innermost corona $C_{1}$, the relationship between deployed $N u$ $m \operatorname{In} C_{i}$ and desireNumInC$C_{i}$ is determined sequentially, and then

Step 3.1. If deployedNumIn $C_{i}>$ desireNumIn $C_{i}$, then deployedNumIn $C_{i}$ - desireNumInC $C_{i}$ nodes nearer to corona $C_{i-1}$ are selected from $C_{i}$ to move straight to $C_{i-1}$. Based on such analysis, the number of sensors deployed in $C_{i-1}$ can be updated as deployedNumIn $C_{i-1}=$

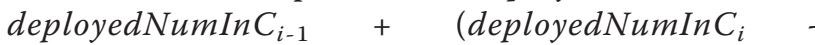
desireNumIn $C_{i}$ );

Step 3.2. If deployedNumIn $C_{i}<$ desireNumIn $C_{i}$, then desireNumIn $C_{i}$ - deployedNumIn $C_{i}$ nodes nearer to corona $C_{i}$ are selected from $C_{i-1}$ to move straight to $C_{i}$. Similarly, the number of sensors in $C_{i-1}$ is updated as deployedNumIn $C_{i-1}=$ deployedNumIn $C_{i-1}-($ desireNu$m \operatorname{In} C_{i}$ - deployedNumIn $C_{i}$ ).

\subsection{Movements control in each corona}

According to Equation (19), the equivalent sensing radius is only related to corona number $i$. Therefore, the movement control in corona $C_{i}$ is similar to the traditional uniform node distribution problem. The main objective of movement control in each corona is to fully cover $C_{i}$ with minimum moving distance.

Define the sensor set in $C_{i}$ as $\operatorname{Set}_{i}=\left\{s_{i 1}, s_{i 2}, \ldots, s_{i n}\right\}$, the initial and final location of $\operatorname{Set}_{i}$ are $L_{i \text {-initial }}=\left\{l_{i 1}, l_{i 2}, \ldots\right.$, $\left.l_{i n}\right\}$ and $L_{i-\text { final }}=\left\{l_{i 1}^{\prime}, l_{i 2}^{\prime}, \ldots, l_{i n}^{\prime}\right\}$. The movements control in $C_{i}$ can be described as the following Multi-objective Optimization Problem (MOP):

$$
\begin{aligned}
& \min \left\{\begin{array}{l}
f_{1}(\bar{x})=1-P_{\text {cov }}\left(S_{i}\right) \\
f_{2}(\bar{x})=\sum_{i \in S_{i}}\left\|l_{i}^{\prime}-l_{i}\right\|
\end{array}\right. \\
& \text { s.t. }\left\|l_{i}^{\prime}-l_{i}\right\| \leq d_{t h} \\
& (i-1) * R_{s} \leq\left\|l_{i}^{\prime}-l_{\sin k}\right\| \leq i * R_{s}
\end{aligned}
$$

Remarks:

- The objective function wants to maximize the network coverage rate while minimize the total moving distance of sensors.

- The first constraint requires that the distances between the initial and final position of any sensor is not larger than $d_{\text {th }}$.

- The second constraint requires that the new location for each sensor is still in the region of corona $C_{i}$.

As discussed above, the goal of movement control in corona is to find the solutions giving the best trade-off between the two conflict objectives, known as Pareto optimal. As NSGA-II is recognized to be well qualified 
to tackle MOPs, we then propose a NSGA-II-based algorithm to find the best node distribution in each corona.

NSGA-II works by evolving a set of solutions to a problem inspired by the genetic mechanisms of natural species evolution [23]. In order to tailor NSGA-II for a particular problem, the individual representation and the corresponding recombination and mutation operator are inevitable.

Represent solutions for the problem is the first issue in an NSGA-II application. Aiming at the problem of node distribution optimization in each corona, an effective coding scheme is applied to represent solutions based on node coordinate, as shown in Figure 3.

The implementation of recombination and mutation necessarily depends on the underlying genotypic representation. As the node coordinate-based representation is adopted, the traditional binary genetic operator cannot be used directly. Therefore, the simulated binary crossover operator (SBX) [24] is applied. Define the parents are $\bar{a}_{1}$ and $\bar{a}_{2}$, and the two children produced by $\mathrm{SBX}$ are $\vec{c}_{1}$ and $\vec{c}_{2}$. In hence, the gene $C_{l, i}\left(x_{c_{1, i}}, \gamma_{c_{1, i}}\right)$ and $C_{2, i}\left(x_{c_{2, i}}, y_{c_{2, i}}\right)$ in children $\vec{c}_{1}$ and $\vec{c}_{1}$ are

$$
\begin{gathered}
\left\{\begin{array}{l}
x_{c_{1, i}}=0.5 \cdot\left((1+\beta) \cdot x_{a_{1, i}}+(1-\beta) \cdot x_{a_{2, i}}\right), \\
y_{c_{1, i}}=0.5 \cdot\left((1+\beta) \cdot y_{a_{1, i}}+(1-\beta) \cdot y_{a_{2, i}}\right) .
\end{array}\right. \\
\left\{\begin{array}{l}
x_{c_{2, i}}=0.5 \cdot\left((1-\beta) \cdot x_{a_{1, i}}+(1+\beta) \cdot x_{a_{2, i}}\right), \\
y_{c_{2, i}}=0.5 \cdot\left((1-\beta) \cdot y_{a_{1, i}}+(1+\beta) \cdot y_{a_{2, i}}\right) .
\end{array}\right.
\end{gathered}
$$

where $\beta$ is the upper limit of the integral corresponding to the probability distribution curve. The mutation operator is applied for each new child generated after recombination. In this article, the exponential mutation is adopted. If an element $a_{i}$ is selected to be mutated, its value changed as

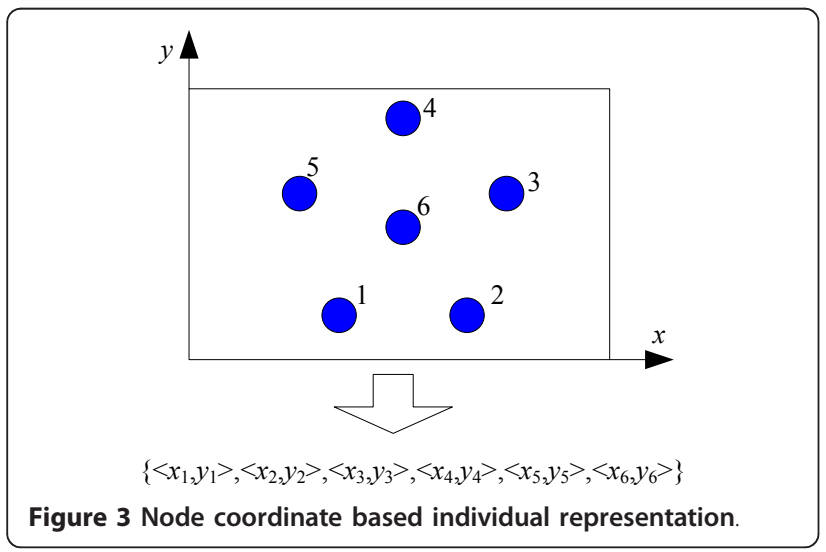

$$
a_{i}^{\prime}=\left\{\begin{array}{l}
a_{i}-\delta\left(t, a_{i}-L_{i}\right), \operatorname{random}(0,1)<0.5 \\
a_{i}+\delta\left(t, U_{i}-a_{i}\right), \operatorname{random}(0,1)>0.5
\end{array}\right.
$$

where $t$ is the generation number, $L_{i}$ and $U_{i}$ are, respectively, the lower and upper bounds of $a_{i}$, which is defined by the second constraint in Equation (26). Note that $\delta(t$, $x$ ) is an exponential function, and it is defined as

$$
\delta(t, x)=x \cdot u \cdot\left(-\frac{t}{T}\right)^{\eta}, \quad 0 \leq u \leq 1
$$

where $u$ is a random number, $T$ is the maximum number of generations, and $\eta$ is an exponent determining the probability distribution.

Similar to the VFA [4], the execution of node distribution problem is designed to be executed on the sink or cluster node, which is expected to have more computational resources. In this way, it would save more computing power for each individual sensor. The sink or the cluster head uses our algorithm to find these appropriate locations, and the designated positions are sent back to the sensors. No movements are performed during the execution of the algorithm. The main procedure of our algorithm is described as follows.

Input: Initial sensor location $\left\{\bar{X}_{S_{-} \text {initial }}, \bar{Y}_{S_{-} \text {initial }}\right\}$ in corona $C_{i}$

The number of generations $T$ and the population size $K$

The recombination probability $P_{r}$;

The mutation probability $P_{m}$;

The reduction rate of controlled elitism $\rho$.

Output: new sensors' location $\left\{\bar{X}_{S}, \bar{Y}_{S}\right\}$ in corona $C_{i}$ Step 1 (initialization):

Set $t=0, P^{\prime}=\varphi$; Generate an initial population $P$ randomly; Calculate $f_{1}(x)$ and $f_{2}(x)$ for each individual by Equation (24);

Step 2 (Non-dominated sorting):

$$
P=P \cup P^{\prime}
$$

Do fast non-dominated sorting algorithm, resulting non-dominated fronts $\left(F_{1}, F_{2}, \ldots, F_{R}\right)$;

Step 3 (controlled elitism)

Set $r=1$ and $P=\varphi$;

While $|P|<K$ do

(1) Calculate $n_{r}$ according to the controlled elitism scheme; 
(2) Sort $F_{r}$ in descending order using crowded comparison;

(3) Put the first $n_{r}$ members of $F_{r}$ in $P$, i.e., $P=P \cup F_{r}$ [1: $\left.n_{r}\right]$;

(4) $r=r+1$.

Step 4 (Fitness assignment):

Assign fitness to each individual according to its position in $P$;

\section{Step 5 (Reproduction)}

Generate an offspring $P$ ' from $P$ according to SBX and mutation operator;

Calculate $f_{1}(x)$ and $f_{2}(x)$ for each individual in $P^{\prime}$;

Step 6 (Termination):

$$
t=t+1
$$

if $t \geq T$ or the required $f_{1}(x)$ and $f_{2}(x)$ are met then terminate;

else go to Step 2.

The complexity of the fast non-dominated sort is $\mathrm{O}$ $\left(2 N^{2}\right)$, the crowding distance assignment is $\mathrm{O}(2 N \log N)$ and the controlled elitism sorting is $\mathrm{O}(2 N \log (2 N))$. Thus, the overall complexity of the above algorithm is $\mathrm{O}\left(2 N^{2}\right)$, where $N$ is the number of sensors deployed in corona $C_{i}$.

\section{Simulation results}

In this section, we will present a set of experiments designed to evaluate the performance of the proposed algorithm. Three metrics, including coverage rate, the total moving distance, and the network survival lifetime, are measured and compared with existing algorithms.

The initial energy reserve of each node $\varepsilon$ is $10000 \mathrm{~J}$, the values of $e_{1}$ and $e_{2}$ are given as follows: $e_{1}=0.5 / 10^{3}$ $\mathrm{J} / \mathrm{bit}, e_{2}=0.25 / 10^{3} \mathrm{~J} / \mathrm{bit}$ [25]. The length of unit sensing data $L$ is 1000 bits, the transmission range of the sensor nodes is 20 , and the sensing radius of each sensor nodes is 8 . The total number of working periods for each sensor is calculated as $\left(\varepsilon-\varepsilon_{\mathrm{red}}\right) /\left[L^{*} M_{i}^{*} e_{1}+L^{*} N_{i} *\left(e_{1}+e_{2}\right)\right]$, where $M_{i}$ is the total number of pixels covered by sensor $s_{i}, N_{i}$ is total number of messages forwarded by $s_{i}$, and $\varepsilon_{\text {red }}$ is the energy consumed in the process of node redistribution, which mainly composes of $\varepsilon_{\text {mov }}$ (energy consumed in mechanical movement) and $\varepsilon_{\text {com }}$ (energy consumed in communication). Here, the energy consumed in movement is normalized into communication. That is, with the same amount of energy consumed in 1 m movement, approximately 300 signal messages can be transmitted [26]. Thus, for any sensor $s_{i}$, the energy consumed in redistribution $\varepsilon_{\text {red }}^{i}$ is

$$
\left\{\begin{array}{l}
\varepsilon_{\mathrm{red}}^{i}=\varepsilon_{\mathrm{mov}}^{i}+\varepsilon_{\mathrm{com}}^{i} \\
\varepsilon_{\mathrm{mov}}^{i}=300 d_{i} \cdot L_{\mathrm{s}} \cdot e_{1} \\
\varepsilon_{\mathrm{com}}^{i}=L_{s} \cdot\left(p_{i} \cdot e_{1}+q_{i} \cdot e_{2}\right)
\end{array}\right.
$$

where $d_{i}$ is the total moving distance, $L_{\mathrm{s}}$ is the length of signal message (set as 100 in this article), $p_{i}$ is the total number of transmitted signal messages, and $q_{i}$ is the total number of received signal messages for redistribution control.

The sensing data forwarding strategy are similar to [16]. As it obeys an approximate uniform distribution in each corona, any node in corona $C_{i}$ can communicate with almost $\rho_{i-1} \cdot A_{i-1} / \rho_{i} \cdot A_{i}$ nodes in the ring $C_{i-1}$ directly. Among these candidate nodes, the node with most residual energy will be selected as the forwarding one.

At first, 64 potential sensors are deployed randomly in the circular area with radius 40, as shown in Figure 4a. The target area is divided into two coronas denoted as

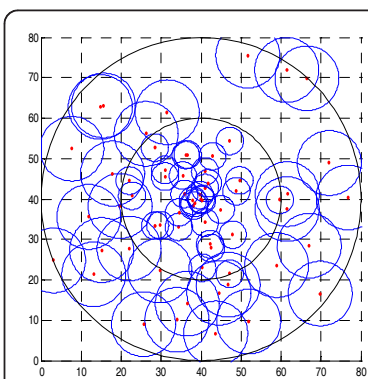

(a) Initial distribution

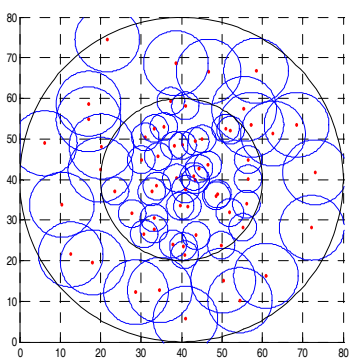

(c) The $30^{\text {th }}$ generation

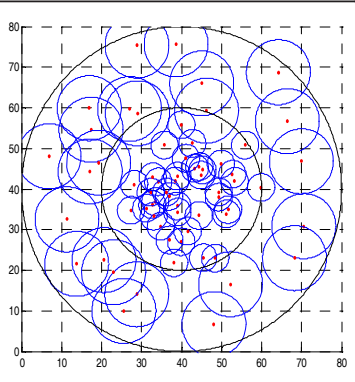

(b) The $10^{\text {th }}$ generation

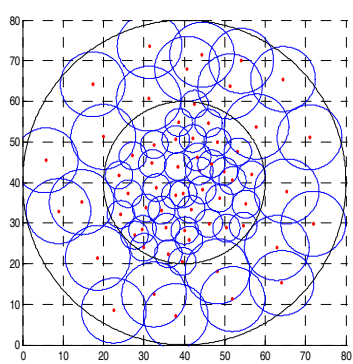

(d) The $100^{\text {th }}$ generation
Figure 4 Illustration of non-dominated solutions obtained in the simulation. (a) Initial distribution, coverage rate $73.25 \%$; (b) the 10th generation, coverage rate $80.53 \%$, total moving distance is 1192 ; (c) the 30th generation, coverage rate $82.43 \%$, total moving distance 1143; (d) the 100th generation, coverage rate $92.43 \%$, total moving distance 1069 
$C_{1}$ and $C_{2}$. From Equation (19), the equivalent sensing radius of $C_{1}$ to $C_{2}$ is calculated as 3.41 and 8 . The population size, the recombination rate, the mutation rate, and the reduction rate are set as 200, 0.9, 0.01, and 0.5, respectively [22]. We compare our algorithm with the VFA approach in terms of the coverage rate, the total moving distance and the network survival time.

The illustration of non-dominated solutions obtained in the simulation is shown in Figure 4. Figure 4a is the initial distribution, and the sensor distribution after running 10 generations is shown in Figure $4 \mathrm{~b}$, in which the coverage rate is $80.53 \%$ and the total mobile distance is 1192. Obviously, much better solutions are obtained in subsequent generations. For instance, compare the solutions in 10th generation with those in 50th generation, the latter uses fewer sensors and achieves a higher coverage rate as shown in Figure 4c. And the solutions shown in Figure 4d are most close to hexagonal geometry generally acknowledged to be the optimal sensor distribution. It achieves the coverage rate of $92.43 \%$ with total moving distance being 1069. In addition, the number of working nodes distributed in corona $C_{1}$ and $C_{2}$ is 24 and 40 , respectively, which is approximate to the energy balance accessibility condition.

In Figure 5, we compare the results of our algorithm with VFA in different number of iterations/generations. Observing the simulation results from the beginning to 200th generation in Figure 5, we can clearly see that our approach directs toward the global optimal solution. And after 100th generation, no better solutions can be found to dominate the sub-optimal solution in our algorithm, which shows that it can converge rapidly to the optimal solution. However, it takes larger total moving distance by using non-uniform distribution strategy. This is mainly due to the smaller equivalent sensing radius in the inner corona. In order to achieve the desired coverage rate, more movement is needed. On the other hand, as our approach has much smaller sensing radius, it helps to mitigate the boundary effects. In conclusion, it gets much higher coverage rate than VFA approach.

Figure 6 shows the comparisons of energy depletion in one working round. As each sensor remembers its sensing pixels, the energy depletion in working rounds does not include the energy consumed in constructing the Voronoi polygon. Here, nodes with smaller ID numbers belong to corona $C_{2}$, and those with larger ID are in corona $C_{1}$. The VFA algorithm are dedicated to achieve a uniform sensor distribution in the target area, so it has a larger distributed density in outer corona and a smaller distributed density in inner corona. As sensors in outer corona only need to transmit its own sensing

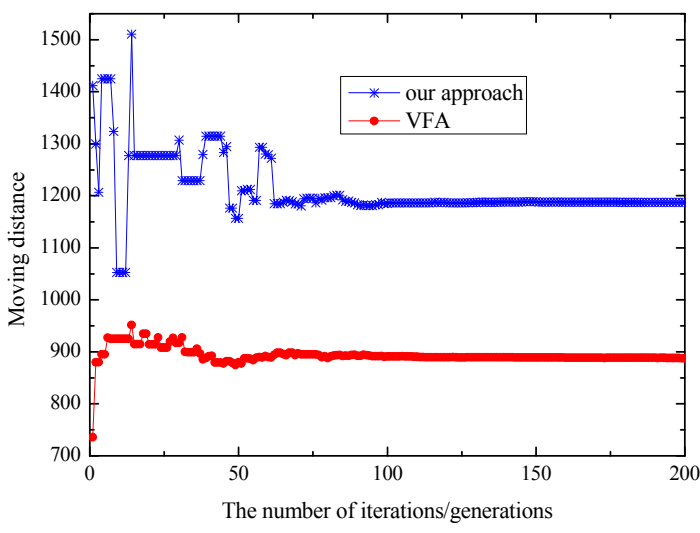

(a) Comparisons of moving distance

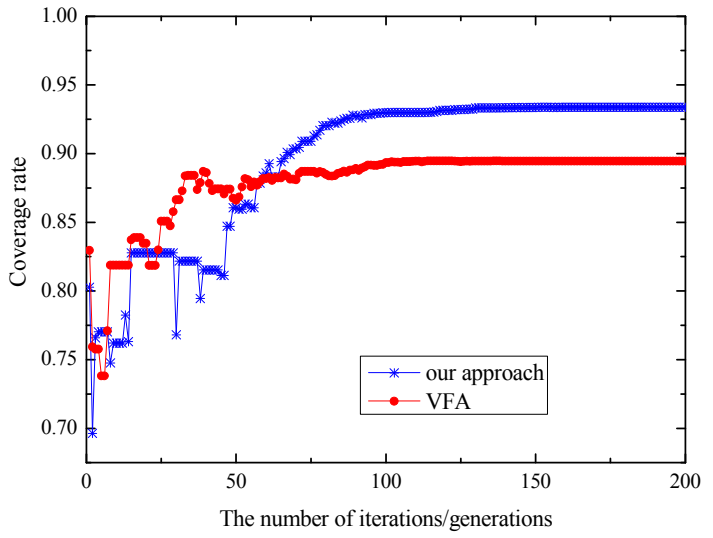

(b) Comparisons of coverage rate

Figure 5 Moving distance and coverage rate comparisons in different number of iterations/generations. (a) Comparisons of moving distance; (b) Comparisons of coverage rate.

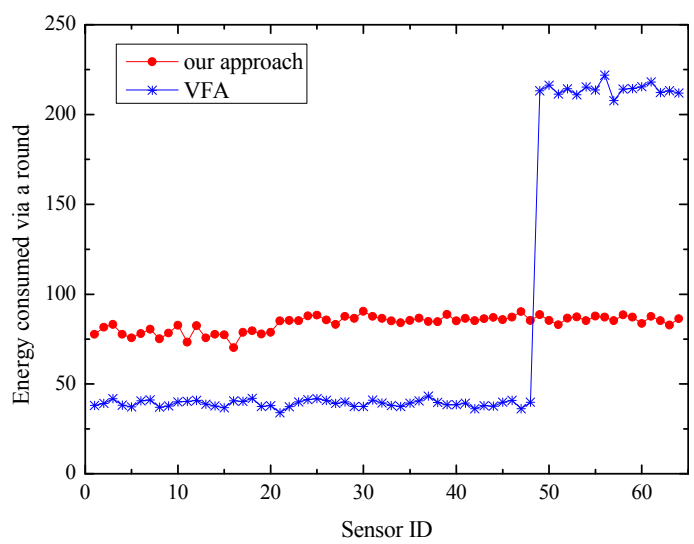

Figure 6 Energy consumption comparisons of each node in a sampling period. 
messages and do not need to forward any other messages, it can consume less energy when the VFA method is used with more sensors in outer corona. Whereas in the inner coronas, those nodes have to receive and forward the messages from outer coronas, the VFA has higher energy depletion than non-uniform approach.

The residual energy of each node at the end of the lifetime is shown in Figure 7. Here, the pixel-based transmission mechanism is also applied in the VFA algorithm. From Figure 7, it is clear to see that the residual energy ratio in non-uniform strategy is much lower than the VFA algorithm. Moreover, the residual energy ratio of each node is nearly the same in non-uniform strategy. In contrast, to the VFA algorithm, while sensors in inner corona are dead, there is more than $85 \%$ energy unused in outmost corona. This is fit to the theoretical expectation and implies the effectiveness of our proposed strategy.

In order to further evaluate the performance of our algorithm, we compare it with VFA and $\delta$-Push\&Pull non-uniform redistribution approach in many cases. There are variable numbers of sensor nodes (varied from 64 to 2675) deployed in different size of target area (varied from 40 to 140). To get the optimal results, all the simulation results are obtained after the genetic algorithm executed more than 500 generations.

Figure 8 shows the comparison of energy consumed in sensor redistribution. As can be seen from Figure 8, when the field area enlarges and the number of nodes increases, our algorithm and $\delta$-Push\&Pull consumed much more energy in the sensor redistribution. This is mainly because that the inner coronas have more sensors deployed than the outer one in non-uniform sensor distribution strategies. The higher density of the inner coronas, the more movement is needed. In $\delta$-Push\&Pull approach, the number of nodes grows in geometric progression from the outer coronas to the inner ones

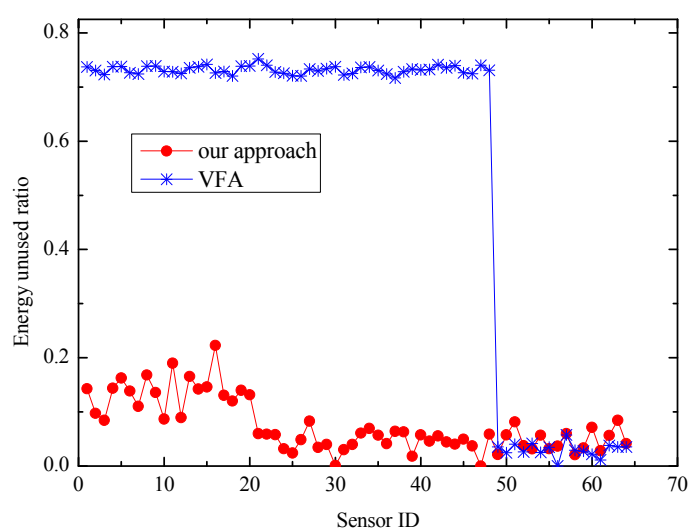

Figure 7 Residual energy comparisons of each node between non-uniform distribution and VFA.

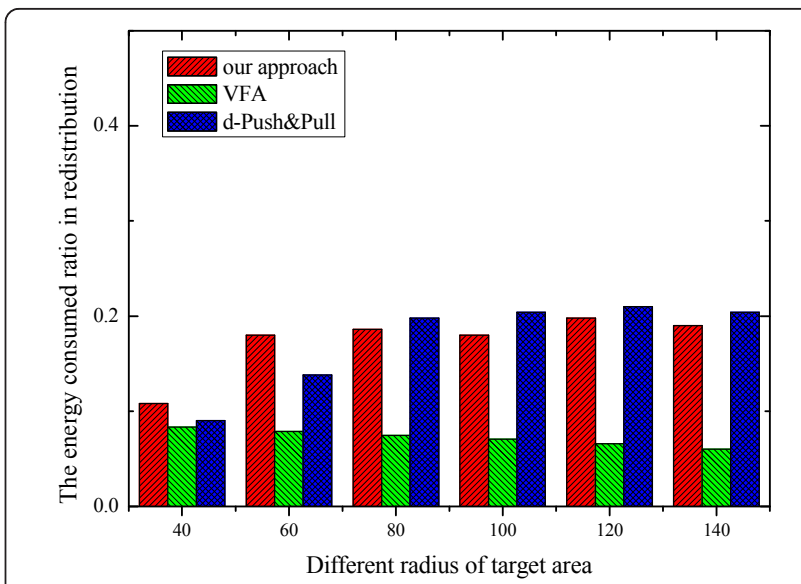

Figure 8 Comparisons of consumed energy ratio in sensor redistribution.

except the outermost corona. Therefore, it has not only more nodes distributed in inner coronas, but also a higher sensor movement than our approach. Compared with non-uniform sensor redistribution, as VFA algorithm merely aims at fully covering the target area, its average moving distance decreases when the node density increases.

Figure 9 shows the comparison of network lifetime in different target area size. Although the uniform distribution aims at maximizing coverage rate with the least sensor movement, it does not consider the imbalance consumption of energy near the sink node. The uneven energy depletion will cause the energy hole and leads to degraded network. When the size of target area increases, the VFA algorithm has the shortest network lifetime, as shown in Figure 9. In addition, although $\delta$ Push\&Pull adopts a non-uniform node distribution, its

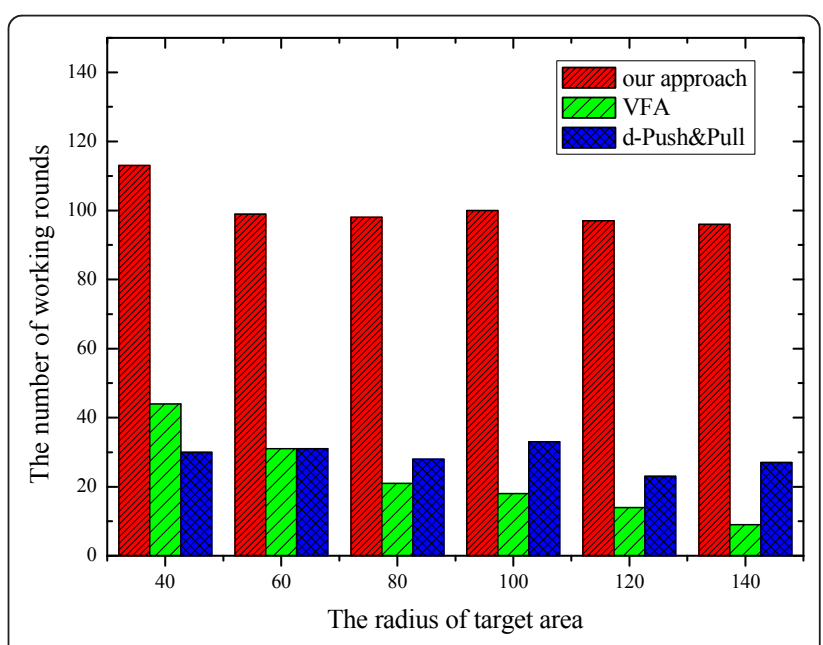

Figure 9 The network lifetime of three algorithms in different target areas. 
network lifetime is not superior to VFA by adopting the pixel-based transmission mechanism, in which the traffic burden caused by constant data acquisition is reduced effectively. By importing the pixel-based transmission mechanism and satisfying the node density for energybalanced depletion condition, our approach has the smallest traffic burden in each working round, and achieves the longest network lifetime.

\section{Conclusion and future work}

In this article, we focus on the problem of sensor redistribution to eliminate energy hole in mobile sensor networks. We present a theoretical analysis of energy attenuation in non-uniform distribution strategy, and prove that when the pixel-based transmission mechanism is adopted, a full energy balance can be achieved through the rational node distribution density. Contributively, we propose a novel non-uniform distribution algorithm with the concept of equivalent sensing radius to achieve energy-balanced depletion while minimizing sensor movement. Simulation results show that our algorithm achieves a better performance than the existing algorithms and can prolong the network lifetime effectively.

In the future, as our study requires that each node knows how to measure its current energy level, we plan to implement our approach in real systems and validate its efficiency in some potential applications such as topology control, distributed storage, and network health monitoring. We also intend to extend our approach to the probabilistic sensing models and 3D space.

\begin{abstract}
Acknowledgements
This study was supported by the National Natural Science Foundation of China under Grant Nos. 60903159, 61173153, 61070162, 71071028, and 70931001; the Specialized Research Fund for the Doctoral Program of Higher Education under Grant No. 20070145017; China Postdoctoral Science Foundation funded project under Grant No. 20110491508; the Fundamental Research Funds for the Central Universities under Grant Nos. N110404014 and N110318001.
\end{abstract}

\section{Competing interests}

The authors declare that they have no competing interests.

Received: 3 October 2011 Accepted: 28 February 2012

Published: 28 February 2012

\section{References}

1. IF Akyildiz, S Weilian, Y Sankarasubramaniam, E Cayirci, A survey on sensor networks. IEEE Commun Mag. 40(8), 102-114 (2002). doi:10.1109/ MCOM.2002.1024422

2. P Basu, J Redi, Movement control algorithms for realization of fault-tolerant ad hoc robot networks. IEEE Netw. 18(4), 36-44 (2004). doi:10.1109/ MNET.2004.1316760

3. N Heo, PK Varshney, Energy-efficient deployment of intelligent mobile sensor networks. IEEE Trans Syst Man Cybern A: Syst Humans. 35(1), 78-92 (2005). doi:10.1109/TSMCA.2004.838486
4. Y Zou, K Chakrabarty, Sensor deployment and target localization based on virtual forces, in Proceedings of the IEEE INFOCOM, vol. 2. (San Francisco, 2003), pp. 1293-1303

5. G Wang, G Cao, TL Porta, W Zhang, Sensor relocation in mobile sensor networks, in Proceedings of the IEEE INFOCOM, vol. 4. (Miami, FL, USA, 2005), pp. 2302-2312

6. PG Wang, G Cao, TL Porta, Movement-assisted sensor deployment. IEEE Transactions on Mobile Computing. 5(6), 640-652 (2006)

7. Z Butler, D Rus, Event-based motion control for mobile sensor networks. IEEE Pervasive Comput. 2(4), 34-42 (2003). doi:10.1109/MPRV.2003.1251167

8. J Wu, S Yang, SMART: a scan-based movement-assisted sensor deployment method in wireless sensor networks, in Proceedings of the IEEE INFOCOM, vol. 4. (Miami, FL, USA, 2005), pp. 2313-2324

9. J Li, P Mohapatra, An analytical model for the energy hole problem in many-to-one sensor networks, Proceedings of the IEEE 62nd Vehicular Technology Conf. (VTC'05), (Dallas, Texas, USA, 2005), pp. 2721-2725

10. J Li, P Mohapatra, Analytical modeling and mitigation techniques for the energy hole problems in sensor networks. Pervasive Mob. Comput. 3(8), 233-254 (2007)

11. C Song, M Liu, J Cao, Y Zheng, H Gong, G Chen, Maximizing network lifetime based on transmission range adjustment in wireless sensor networks. Comput. Commun. 32(11), 1316-1325 (2009). doi:10.1016/j. comcom.2009.02.002

12. HY Shiue, GJ Yu, JP Sheu, Energy hole healing protocol for surveillance sensor networks, Workshop on WASN, (Taiwan, China, 2005)

13. W Wang, $V$ Srinivasan, $K$ Chua, Using mobile relays to prolong the lifetime of wireless sensor networks, Proceedings of the ACM MobiCom'05, (Cologne, Germany, 2005), pp. 270-283

14. J Luo, JP Hubaux, Joint mobility and routing for lifetime elongation in wireless sensor networks, in Proceedings of the IEEE INFOCOM'05, vol. 3. (Miami, FL, USA, 2005), pp. 1735-1746

15. S Olariu, I Stojmenovic, Design guidelines for maximizing lifetime and avoiding energy holes in sensor networks with uniform distribution and uniform reporting, Proceedings of the IEEE INFOCOM, (Barcelona, Catalunya, Spain, 2006), pp. 1-12

16. $\mathrm{X}$ Wu, G Chen, SK Das, On the energy hole problem of nonuniform node distribution in wireless sensor networks. IEEE Trans. Parallel Distrib. Syst. 19(5), 710-720 (2008)

17. N Bartolini, T Calamoneri, A Massini, S Silvestri, On adaptive density deployment to mitigate the sink-hole problem in mobile sensor networks. ACM/Springer Mob. Netw. Appl. 16(1), 134-145 (2011). doi:10.1007/s11036010-0247-5

18. F Aurenhammer, Voronoi diagrams: a survey of a fundamental geometric data structure. ACM Comput. Surv. 23, 345-405 (1991). doi:10.1145/ 116873.116880

19. S Fortune, Voronoi diagrams and delaunay triangulations, Computing in Euclidean Geometry, (World Scientific Publishing Co., Singapore, 1992), pp. 193-223

20. K Deb, A Pratap, S Agarwal, T Meyarivan, A fast and elitist multi-objective genetic algorithm: NSGA-II. IEEE Trans Evolution Comput. 6(2), 182-197 (2002). doi:10.1109/4235.996017

21. H Zhang, JC Hou, Maintaining sensing coverage and connectivity in large sensor networks. Ad Hoc Wirel Netw. 1(1), 89-124 (2005)

22. J Jia, J Chen, G Chang, Z Tan, Energy efficient coverage control in wireless sensor networks based on multi-objective genetic algorithm. Comput. Math. Appl. 57(11), 1756-1766 (2009). doi:10.1016/j.camwa.2008.10.036

23. DE Goldberg, Genetic Algorithms in Search, Optimization and Machine Learning, (Addison-Wesley Longman Publishing Co., Boston, MA, USA, 1989)

24. K Deb, RB Agarwal RB, Simulated binary crossover for continuous search space. Complex Syst. 9(2), 115-148 (1995)

25. G Anastasi, M Conti, A Falchi, E Gregori, A Passarella, Performance measurements of mote sensor networks, Proceedings of the ACM MSWiM, (Venice, Italy, 2004), pp. 174-181

26. GT Sibley, MH Rahimi, GS Sukhatme, Robomote: a tiny mobile robot platform for large-scale sensor networks, Proceedings of the IEEE Int'l Conf. Robotics and Automation, (Washington, DC, USA, 2002), pp. 1143-1148

doi:10.1186/1687-1499-2012-68

Cite this article as: Jia et al:: Exploiting sensor redistribution for eliminating the energy hole problem in mobile sensor networks. EURASIP Journal on Wireless Communications and Networking 2012 2012:68. 